\title{
Lojasiewicz exponents, the integral closure of ideals and Newton polyhedra
}

\author{
By Carles Bivì̀-AusinA
}

(Received Apr. 13, 2001)

(Revised Jan. 15, 2002)

\begin{abstract}
We give an upper estimate for the Lojasiewicz exponent $\ell(J, I)$ of an ideal $J \subseteq A\left(\boldsymbol{K}^{n}\right)$ with respect to another ideal $I$ in the ring $A\left(\boldsymbol{K}^{n}\right)$ of germs analytic functions $f:\left(\boldsymbol{K}^{n}, 0\right) \rightarrow \boldsymbol{K}$, where $\boldsymbol{K}=\boldsymbol{C}$ or $\boldsymbol{R}$, using Newton polyhedrons. In particular, we give a method to estimate the Lojasiewicz exponent $\alpha_{0}(f)$ of a germ $f \in A\left(\boldsymbol{K}^{n}\right)$ that can be applied when $f$ is Newton degenerate with respect to its Newton polyhedron.
\end{abstract}

\section{Introduction.}

The technique of using Newton polyhedrons has many significant applications in singularity theory, as can be seen in the works of Fukui [5], Kouchnirenko [8], Oka [13], Varchenko [17], Yoshinaga [18] and other authors. In this article we are interested in using Newton polyhedrons in order to give an upper estimate of the Lojasiewicz exponent $\ell(J, I)$ of an ideal $J \subseteq A\left(\boldsymbol{K}^{n}\right)$ with respect to another ideal $I \subseteq A\left(\boldsymbol{K}^{n}\right)$ (see Definition 2.1), where $A\left(\boldsymbol{K}^{n}\right)$ is the local ring of analytic function germs $f:\left(\boldsymbol{K}^{n}, 0\right) \rightarrow \boldsymbol{K}$ and $\boldsymbol{K}=\boldsymbol{R}$ or $\boldsymbol{C}$. As we shall see, this estimation is possible when $I$ admits a generating system which is adapted to a Newton polyhedron, which is a notion introduced in this paper. This class of systems contains the one defined by Fukui, Saia and the author in [1] in order to compute multiplicities using the information given by Newton filtrations. Therefore, ideals generated by adapted systems is a notion generalizing the ideals generated by the components of a semi-weighted-homogeneous map germ.

The result of Lejeune-Teissier [10] characterizing the integral closure of an ideal in $A\left(\boldsymbol{C}^{n}\right)$ using a Łojasiewicz type inequality motivates the definition of the ideals $\overline{I^{\theta}}$, where $\theta$ is a positive rational number and $I$ is an ideal of $A\left(\boldsymbol{K}^{n}\right)$ (see Section 2). The main result of this work (Theorem 3.5) gives a sufficient condition for a monomial $x^{k}=x_{1}^{k_{1}} \cdots x_{n}^{k_{n}}$ to belong to an ideal $\overline{I^{\theta}}$, where $\theta$ is a positive rational number. The mentioned result on the estimation of Lojasiewicz exponents is seen as a consequence of this result. Moreover, taking $\theta=1$, we have an approximation to the Newton polyhedron determined by the monomials belonging to the integral closure of $I$.

If $f:\left(\boldsymbol{K}^{n}, 0\right) \rightarrow \boldsymbol{K}$ is an analytic function germ with an isolated singularity at the origin, we can consider the Lojasiewicz exponent $\ell\left(m_{n}, J(f)\right)$, where $J(f)$ is the jacobian ideal of $f$ and $m_{n}$ the maximal ideal of $A\left(\boldsymbol{K}^{n}\right)$. This number, also denoted by $\alpha_{0}(f)$, is the least upper bound of those $\alpha>0$ such that

2000 Mathematics Subject Classification. Primary 32S05; Secondary 58A20.

Key Words and Phrases. Lojasiewicz exponents, real analytic functions, Newton polyhedrons.

Work supported by DGICYT Grant BFM2000-1110. 


$$
|x|^{\alpha} \leq C\left|\left(\frac{\partial f}{\partial x_{1}}, \ldots, \frac{\partial f}{\partial x_{n}}\right)\right|
$$

for some constant $C>0$ and all $x$ in an open neighbourhood of $0 \in \boldsymbol{K}^{n}$. An upper bound for $\alpha_{0}(f)$ was given by Fukui [5] when $f$ is Newton non-degenerate in the sense of Kouchnirenko [8]. We establish the notion of strongly adapted system in order to give an upper estimate of $\alpha_{0}(f)$. As we shall see in some examples, this estimation can be given when the function $f$ is Newton degenerate. In particular, we are dealing with the problem of computing the order of $C^{0}$-determinacy of analytic function germs $f:\left(\boldsymbol{K}^{n}, 0\right) \rightarrow(\boldsymbol{K}, 0)$, by virtue of the works of Chang-Lu [3] (when $\boldsymbol{K}=\boldsymbol{C}$ ) and Kuo [9] (when $\boldsymbol{K}=\boldsymbol{R}$ ). The number $\alpha_{0}(f)$ is related with other relevant Lojasiewicz exponents attached to $f$, as is shown in [7].

\section{Basic definitions.}

If $S \subseteq A\left(\boldsymbol{K}^{n}\right)$, we denote by $V(S)$ the zero set germ at 0 of $S$ in $\boldsymbol{K}^{n}$.

Definition 2.1. Let $I$ be an ideal of $A\left(\boldsymbol{K}^{n}\right)$ and $h \in A\left(\boldsymbol{K}^{n}\right)$ such that $V(I) \subseteq V(h)$. Let $g_{1}, \ldots, g_{s}$ be a system of generators of $I$. By [12, p. 136] (see also [2, p. 493]), we can consider the greatest lower bound of those $\theta>0$ such that there exists an open neighbourhood $U$ of 0 in $\boldsymbol{K}^{n}$ and a constant $C>0$ such that

$$
|h(x)|^{\theta} \leq C \sup _{i}\left|g_{i}(x)\right|
$$

for all $x \in U$. We call this number the Eojasiewicz exponent of $h$ with respect to $I$ and we denote it by $\ell(h, I)$. It is easy to see that this definition does not depend on the chosen system of generators of $I$. If $J=\left\langle h_{1}, \ldots, h_{t}\right\rangle \subseteq A\left(\boldsymbol{K}^{n}\right)$ is any other ideal such that $V(I) \subseteq V(J)$, then we set $\ell(J, I)=\max _{i} \ell\left(h_{i}, I\right)$.

By the work [10] of Lejeune-Teissier, if $I \subseteq A\left(\boldsymbol{C}^{n}\right)$ then $\ell(h, I)$ is a rational number. This is also true when $I \subseteq A\left(\boldsymbol{R}^{n}\right)$ by the work of Bochnak-Risler [2].

When $\ell(h, I) \leq 1$, then we will say that $h$ is integral over $I$ (see [6, p. 318]). The set of elements which are integral over $I$ is an ideal of $A\left(\boldsymbol{K}^{n}\right)$ called the integral closure of I. This ideal is denoted by $\bar{I}$ and obviously the inclusion $I \subseteq \bar{I}$ holds. Moreover, if $\theta$ is a positive rational number, we define $\overline{I^{\theta}}$ as the ideal generated by those $h \in A\left(\boldsymbol{K}^{n}\right)$ such that $\ell(h, I) \leq 1 / \theta$. Then, we can express $\ell(h, I)$ as

$$
\ell(h, I)=\frac{1}{\max \left\{\theta \in Q_{+}: h \in \overline{I^{\theta}}\right\}} .
$$

As can be seen in [10, p. 10] or [16, p. 289], if $I$ is an ideal of $A\left(\boldsymbol{C}^{n}\right)$ and $h \in A\left(\boldsymbol{C}^{n}\right)$ is such that $V(I) \subseteq V(h)$, then $h \in \bar{I}$ if and only if $h$ satisfies a relation of the form $h^{m}+a_{1} h^{m-1}+\cdots+a_{m-1} h+a_{m}=0$ for some $m \geq 1$ and some $a_{i} \in I^{i}, i=1, \ldots, m$.

REMARK 2.2. If $I=\left\langle g_{1}, \ldots, g_{s}\right\rangle$ is an ideal of $A\left(\boldsymbol{K}^{n}\right)$ and $g \in \bar{I}$, then it is clear that $\ell(h, I)=\ell\left(h,\left\langle g, g_{1}, \ldots, g_{s}\right\rangle\right)$.

The following is an interesting characterization of the integral closure of an ideal in $A\left(\boldsymbol{K}^{n}\right)$ that we shall use in Section 6. 
Proposition 2.3 ([6, p. 318]). If $I$ is an ideal of $A\left(\boldsymbol{K}^{n}\right)$ and $h \in A\left(\boldsymbol{K}^{n}\right)$ is such that $V(I) \subseteq V(h)$, then $h \in \bar{I}$ if and only if, for each analytic map germ $\varphi:(\boldsymbol{K}, 0) \rightarrow\left(\boldsymbol{K}^{n}, 0\right)$ we have that $\varphi^{*}(h) \in \varphi^{*}(I) A(\boldsymbol{K})$, where $\varphi^{*}: A\left(\boldsymbol{K}^{n}\right) \rightarrow A(\boldsymbol{K})$ is the natural morphism induced by $\varphi^{*}$.

Newton polyhedrons have been already used in the problem of computing the integral closure of an ideal. The first step in this fact is the result stating that the integral closure of a monomial ideal $I \subseteq A\left(\boldsymbol{C}^{n}\right)$ is generated by all the monomials $x^{k}$ such that $k$ belongs to the Newton polyhedron of $I$ (see [4, p. 141] and [15]). The same conclusion was obtained by Yoshinaga in [18] for ideals of the form $I=$ $\left\langle x_{1} \partial f / \partial x_{1}, \ldots, x_{n} \partial f / \partial x_{n}\right\rangle \subseteq A\left(\boldsymbol{K}^{n}\right)$, where $f \in A\left(\boldsymbol{K}^{n}\right)$ is a Newton non-degenerate function in the sense of Kouchnirenko [8]. This result has been extended for a wider class of ideals by Saia [14] thus leading to the notion of Newton non-degenerate ideal. As a consequence of our study, we shall also continue the trajectory of results in this direction introducing a new class of ideals for which we give an approximation of the monomial zone of the integral closure of $I$, that is, the Newton polyhedron determined by the monomials $x^{k}$ such that $x^{k} \in \bar{I}$ (consider the case $\theta=1$ in Theorem 3.5).

We now introduce the notion of Newton polyhedron. We set $\boldsymbol{R}_{+}=\{x \in \boldsymbol{R}: x \geq 0\}$, $\boldsymbol{Q}_{+}=\boldsymbol{Q} \cap \boldsymbol{R}_{+}$and $\boldsymbol{Z}_{+}=\boldsymbol{Z} \cap \boldsymbol{R}_{+}$. If $x_{1}, \ldots, x_{n}$ is a coordinate system in $\boldsymbol{K}^{n}$, we denote by $x^{k}$ the monomial $x_{1}^{k_{1}} \cdots x_{n}^{k_{n}}$, for any $k=\left(k_{1}, \ldots, k_{n}\right) \in \boldsymbol{Z}_{+}^{n}$.

Definition 2.4. Let $\Gamma_{+} \subseteq \boldsymbol{R}_{+}^{n}$, we say that $\Gamma_{+}$is a Newton polyhedron when there exists a subset $A \subseteq \boldsymbol{Q}_{+}^{n}$ such that $\Gamma_{+}$is equal to the convex hull of $\left\{k+v: k \in A, v \in \boldsymbol{R}_{+}^{n}\right\}$. The set $\Gamma_{+}$will also be called the Newton polyhedron determined by $A$ and it is denoted by $\Gamma_{+}(A)$.

Let $g \in A\left(\boldsymbol{K}^{n}\right)$ and $g(x)=\sum_{k} a_{k} x^{k}$ the Taylor development of $g$ around the origin. We define the support of $g$ as $\operatorname{supp}(g)=\left\{k \in \boldsymbol{Z}_{+}^{n}: a_{k} \neq 0\right\}$. Given any set $S \subseteq A\left(\boldsymbol{K}^{n}\right)$, the support of $S$, denoted by $\operatorname{supp}(S)$, is the union of the supports of the elements belonging to $S$. The Newton polyhedron of $S$ is defined as $\Gamma_{+}(S)=\Gamma_{+}(\operatorname{supp}(S))$.

If $I$ is any ideal of $A\left(K^{n}\right)$, it is easy to check that $\Gamma_{+}(I)$ is equal to the convex hull of $\Gamma_{+}\left(g_{1}\right) \cup \cdots \cup \Gamma_{+}\left(g_{s}\right)$, where $g_{1}, \ldots, g_{s}$ is any generating system of $I$.

Following the same proof of Lemma 3.3 of [14], which is based on Proposition 2.3, it follows that $\Gamma_{+}(I)=\Gamma_{+}(\bar{I})$, for any ideal $I \subseteq A\left(\boldsymbol{K}^{n}\right)$.

\section{Adapted systems.}

We denote by $\langle u, v\rangle$ the usual scalar product of two vectors $u, v \in \boldsymbol{R}^{n}$. If $\Gamma_{+}$is a fixed Newton polyhedron and $v \in \boldsymbol{R}_{+}^{n} \backslash\{0\}$, then we define $\ell\left(v, \Gamma_{+}\right)=\min \left\{\langle k, v\rangle: k \in \Gamma_{+}\right\}$ and $\Delta\left(v, \Gamma_{+}\right)=\left\{k \in \Gamma_{+}:\langle k, v\rangle=\ell\left(v, \Gamma_{+}\right)\right\}$. We say that a subset $\Delta \subseteq \Gamma_{+}$is a face of $\Gamma_{+}$when there exists some vector $v \in \boldsymbol{R}_{+}^{n} \backslash\{0\}$ such that $\Delta=\Delta\left(v, \Gamma_{+}\right)$. In this case, we say that $v$ supports $\Delta$. It is easy to see that $\Delta\left(v, \Gamma_{+}\right)$is compact if and only if $v_{i} \neq 0$, for all $i=1, \ldots, n$, where $v=\left(v_{1}, \ldots, v_{n}\right)$. We denote by $\Gamma$ the union of all compact faces of $\Gamma_{+}$.

A vector $v \in \boldsymbol{Z}_{+}^{n} \backslash\{0\}$ is said to be primitive when it is the vector with smallest length over all the vectors in the set $\left\{\lambda v: \lambda \in \boldsymbol{R}_{+}\right\} \cap\left(\boldsymbol{Z}_{+}^{n} \backslash\{0\}\right)$. Then, every face of dimension $n-1$ of a given Newton polyhedron $\Gamma_{+} \subseteq \boldsymbol{R}^{n}$ is supported by a unique primitive vector. 
Given a vector $v \in \boldsymbol{R}_{+}^{n} \backslash\{0\}$ and a series $g=\sum_{k} a_{k} x^{k} \in A\left(\boldsymbol{K}^{n}\right)$, we define $\ell(v, g)=$ $\ell\left(v, \Gamma_{+}(g)\right)$ and $\Delta(v, g)=\Delta\left(v, \Gamma_{+}(g)\right) \cap \operatorname{supp}(g)$.

Let $\Gamma_{+} \subseteq \boldsymbol{R}^{n}$ be a fixed Newton polyhedron. Suppose that the number of $(n-1)$ dimensional faces of $\Gamma_{+}$is $r$. Let $v^{1}, \ldots, v^{r}$ be the primitive vectors of $\Gamma_{+}$supporting some face of dimension $n-1$ of $\Gamma_{+}$.

Definition 3.1. Let $g=\sum_{k} a_{k} x^{k} \in A\left(\boldsymbol{K}^{n}\right)$ and let $J \subseteq\{1, \ldots, r\}$, then we can consider the set $\Delta(J, g)=\bigcap_{j \in J} \Delta\left(v^{j}, g\right)$. We define the principal part of $g$ with respect to $J$ as the polynomial $q_{J}(g)$ given by the sum of those $a_{k} x^{k}$ such that $k \in \Delta(J, g)$. The set $\Delta(J, g)$ could be empty, in this case, we fix $q_{J}(g)=0$.

Definition 3.2. Given $S=\left\{g_{1}, \ldots, g_{s}\right\} \subseteq A\left(\boldsymbol{K}^{n}\right)$, we say that $S$ is an adapted system to $\Gamma_{+}$(or that $S$ is $\boldsymbol{K}$-adapted to $\Gamma_{+}$) when, for each $J \subseteq\{1, \ldots, r\}$ such that $\bigcap_{j \in J} \Delta\left(v^{j}, \Gamma_{+}\right)$is a compact face of $\Gamma_{+}$, the system of equations

$$
q_{J}\left(g_{1}\right)(x)=\cdots=q_{J}\left(g_{s}\right)(x)=0,
$$

has no solution in $(\boldsymbol{K} \backslash\{0\})^{n}$. We say that $S$ is an adapted system when $S$ is adapted to the Newton polyhedron determined by $S$.

EXAmple 3.3. Let $S=\left\{x^{3} y+x^{7}, x y^{3}+y^{7}\right\} \subseteq A\left(\boldsymbol{R}^{2}\right)$. Then, after some elementary computations, it follows that $S$ is an adapted system. An example of a non adapted system of $A\left(\boldsymbol{R}^{2}\right)$ is $S^{\prime}=\left\{x^{3} y+x^{7}-x^{2} y^{2}, x y^{3}+y^{7}-x^{2} y^{2}\right\}$.

REMARK 3.4. i) When we consider an $\boldsymbol{R}$-adapted system $S=\left\{g_{1}, \ldots, g_{s}\right\} \subseteq A\left(\boldsymbol{R}^{n}\right)$ to $\Gamma_{+}$then, complexifying the variables, we could obtain another system in $A\left(\boldsymbol{C}^{n}\right)$ which is not $\boldsymbol{C}$-adapted to $\Gamma_{+}$in general. Unless some risk of confusion appears, we will not specify which ground field we are dealing with.

ii) If some element of the system $S=\left\{g_{1}, \ldots, g_{s}\right\} \subseteq A\left(\boldsymbol{K}^{n}\right)$ is a monomial $x^{k}=$ $x_{1}^{k_{1}} \cdots x_{n}^{k_{n}}$, then $S$ is adapted to any Newton polyhedron.

iii) It is straightforward to check that, if $S=\left\{g_{1}, \ldots, g_{s}\right\} \subseteq A\left(\boldsymbol{C}^{n}\right)$ is a nondegenerate system with respect to a Newton polyhedron $\Gamma_{+} \subseteq \boldsymbol{R}_{+}^{n}$ in the sense of [1]], then $S$ is also adapted to $\Gamma_{+}$(the system $S$ of Example 3.3 shows that the converse is not true).

Now, we state the main result of this work, which will be proved in the next section.

THEOREM 3.5. Let $\Gamma_{+} \subseteq \boldsymbol{R}^{n}$ be a Newton polyhedron and let $S=\left\{g_{1}, \ldots, g_{s}\right\} \subseteq$ $A\left(\boldsymbol{K}^{n}\right)$ be an adapted system to $\Gamma_{+}$. Let $I \subseteq A\left(\boldsymbol{K}^{n}\right)$ be the ideal generated by $S$ and let $\theta \in \boldsymbol{Q}_{+}$. Suppose that $x^{k}$ is a monomial such that $\left\langle k, v^{j}\right\rangle \geq \theta \max \left\{\ell\left(v^{j}, g_{1}\right), \ldots, \ell\left(v^{j}, g_{s}\right)\right\}$, for all $j=1, \ldots, r$. Then $x^{k} \in \overline{I^{\theta}}$.

\section{The proof of Theorem 3.5.}

Along this section, we will suppose that $\Gamma_{+}$is a Newton polyhedron contained in $\boldsymbol{R}^{n}$. As in the previous section, we denote by $r$ the number of $(n-1)$-dimensional faces of $\Gamma_{+}$and we also denote by $v^{1}, \ldots, v^{r}$ the primitive vectors supporting some $(n-1)$-dimensional face of $\Gamma_{+}$. 
We consider the equivalence relation in $\boldsymbol{R}_{+}^{n} \backslash\{0\}$ given by $v \sim v^{\prime}$ if and only if $\Delta(v)=\Delta\left(v^{\prime}\right)$. Let $\Gamma^{*}$ denote the corresponding quotient space. Each equivalence class can be identified with a convex cone with vertex at the origin $\sigma=\boldsymbol{R}_{+} a^{1}(\sigma)+\cdots+$ $\boldsymbol{R}_{+} a^{s}(\sigma)$, where we suppose that $a^{1}(\sigma), \ldots, a^{s}(\sigma)$ are primitive vectors (see [18, p. 810]). When the vectors $a^{1}(\sigma), \ldots, a^{s}(\sigma)$ are linearly independent over $\boldsymbol{R}$, then $\sigma$ is said to be a simplicial cone. A face of $\sigma$ is any cone $\sigma^{\prime}$ contained in the boundary of $\sigma$ and spanned by a subset of $\left\{a^{1}(\sigma), \ldots, a^{s}(\sigma)\right\}$.

We denote by $\Sigma_{0}$ the collection of cones arising from the equivalence classes of $\Gamma^{*}$. This collection can be considered as a subdivision of $\boldsymbol{R}_{+}^{n}$. Given a cone $\sigma \in \Sigma_{0}$, we say that $\sigma$ has dimension $d$ when the smallest vector subspace containing $\sigma$ has dimension $d$, where $d \in\{1, \ldots, n\}$.

Suppose we are given a $d$-dimensional cone $\sigma \in \Sigma_{0}$ and that this is written in the form $\sigma=\boldsymbol{R}_{+} a^{1}(\sigma)+\cdots+\boldsymbol{R}_{+} a^{s}(\sigma)$, for some primitive vectors $a^{1}(\sigma), \ldots, a^{s}(\sigma), s \geq d$. Since $\sigma$ is $d$-dimensional, we can suppose that $a^{1}(\sigma), \ldots, a^{d}(\sigma)$ are linearly independent, by Carathéodory's theorem (see [4, p. 139]). Let $\tau$ be the cone spanned by $a^{1}(\sigma), \ldots, a^{d}(\sigma)$. If $\overline{\sigma \backslash \tau}$ is a simplicial cone, then we replace the cone $\sigma$ in $\Sigma_{0}$ by the pair $\tau, \overline{\sigma \backslash \tau}$ and all the faces of $\tau$ and $\overline{\sigma \backslash \tau}$ not yet included in $\Sigma_{0}$, where we denote by $\bar{A}$ the closure of a subset $A \subseteq \boldsymbol{R}^{n}$. Otherwise, we apply again Carathéodory's theorem to the closure of each connected component of $\sigma \backslash \tau$ and proceed in the same way. Therefore, from the collection of cones $\Sigma_{0}$ we obtain, by a simple inductive process, a collection of cones $\Sigma$ such that each cone $\sigma \in \Sigma$ is simplicial and the set of 1-dimensional cones of $\Sigma$ is equal to the set of 1-dimensional cones of $\Sigma_{0}$.

REMARK 4.1. If $\Gamma_{+}$is a Newton polyhedron in $\boldsymbol{R}^{2}$ then, the subdivision $\Sigma$ can be taken equal to $\Sigma_{0}$, since all cones belonging to $\Sigma_{0}$ are simplicial cones of dimension 1 and 2 in this case.

We denote the family of $d$-dimensional cones of $\Sigma$ by $\Sigma^{(d)}, d=1, \ldots, n$. Attached to each $\sigma \in \Sigma^{(n)}$, we will consider the primitive vectors $a^{1}(\sigma), \ldots, a^{n}(\sigma)$ arising from the simplicial structure of $\sigma$. We choose an order of these vectors that will be fixed throughout this section. Moreover, we write $a^{i}(\sigma)=\left(a_{1}^{i}(\sigma), \ldots, a_{n}^{i}(\sigma)\right)$, for all $i=$ $1, \ldots, n$.

For each $\sigma \in \Sigma^{(n)}$, let $\boldsymbol{K}^{n}(\sigma)$ be a copy of $\boldsymbol{K}^{n}$ and let $\left(y_{\sigma, 1}, \ldots, y_{\sigma, n}\right)$ be a system of coordinates in $\boldsymbol{K}^{n}(\sigma)$. We define $W_{\sigma}^{C}=\left\{y_{\sigma} \in \boldsymbol{C}^{n}(\sigma):\left|y_{\sigma, j}\right| \leq 1, \forall j=1, \ldots, n\right\}$ and $V^{C}=\left\{x \in C^{n}:\left|x_{j}\right| \leq 1, \forall j=1, \ldots, n\right\}$. Then, we consider the map $\pi_{\sigma}^{C}: W_{\sigma}^{C} \rightarrow V^{C}$ defined as

$$
\pi_{\sigma}^{C}\left(y_{\sigma, 1}, \ldots, y_{\sigma, n}\right)=\left(y_{\sigma, 1}^{a_{1}^{1}(\sigma)} \cdots y_{\sigma, n}^{a_{1}^{n}(\sigma)}, \ldots, y_{\sigma, 1}^{a_{n}^{1}(\sigma)} \cdots y_{\sigma, n}^{a_{n}^{n}(\sigma)}\right) .
$$

Let $W_{\Sigma}^{C}$ be the disjoint union of the sets $W_{\sigma}^{C}$, where $\sigma \in \Sigma^{(n)}$, and let $\pi^{C}: W_{\Sigma}^{C} \rightarrow V^{C}$ be the map given by $\pi^{C}\left(y_{\sigma}\right)=\pi_{\sigma}^{C}\left(y_{\sigma}\right)$, for all $y_{\sigma} \in W_{\sigma}^{C}$. We endow $W_{\Sigma}^{C}$ with the natural topology. Given a cone $\sigma \in \Sigma^{(n)}$, we also define $W_{\sigma}^{\boldsymbol{R}}=\left(\pi_{\sigma}^{\boldsymbol{C}}\right)^{-1}\left(V^{\boldsymbol{R}}\right) \subseteq$ $\boldsymbol{C}^{n}(\sigma)$, where $V^{\boldsymbol{R}}=V^{\boldsymbol{C}} \cap \boldsymbol{R}^{n}$. Then, we denote by $W_{\Sigma}^{\boldsymbol{R}}$ the disjoint union of the sets $W_{\sigma}^{\boldsymbol{R}}$, where $\sigma \in \Sigma^{(n)}$, and we define the map $\pi^{\boldsymbol{R}}: W_{\Sigma}^{\boldsymbol{R}} \rightarrow V^{\boldsymbol{R}}$ as the restriction of $\pi^{\boldsymbol{C}}$ to $W_{\Sigma}^{R}$.

Lemma 4.2. The map $\pi^{\boldsymbol{K}}: W_{\Sigma}^{\boldsymbol{K}} \rightarrow V^{\boldsymbol{K}}$ is proper and surjective, for $\boldsymbol{K}=\boldsymbol{C}$ or $\boldsymbol{R}$. 
Proof. The proof of the case $\boldsymbol{K}=\boldsymbol{C}$ is the same as the one of [5, Lemma 4.9]. The case $\boldsymbol{K}=\boldsymbol{R}$ follows from the complex case and the definition of the sets $W_{\sigma}^{\boldsymbol{R}}$, $\sigma \in \Sigma^{(n)}$.

When there is no risk of confusion, we shall delete the superscripts from $W_{\sigma}^{\boldsymbol{K}}, W_{\Sigma}^{\boldsymbol{K}}$, $\pi_{\sigma}^{\boldsymbol{K}}, \pi^{\boldsymbol{K}}$ and $V^{\boldsymbol{K}}$. We will also need the following auxiliary lemma from elementary topology in the proof of Theorem 3.5.

Lemma 4.3. Let $X$ and $Y$ be topological spaces, where $Y$ is locally compact and Hausdorff, let $f: X \rightarrow Y$ a proper map and let $p \in Y$. Then, for any open set $A$ of $X$ such that $f^{-1}(p) \subseteq A$, there exists an open set $B$ in $Y$ such that $p \in B$ and $f^{-1}(B) \subseteq A$.

Proof. Since $Y$ is locally compact and Hausdorff, we can consider a base of compact neighbourhoods $\left\{A_{n}\right\}_{n \geq 1}$ of $p$ such that $\bigcap_{n \geq 1} A_{n}=\{p\}$ and $A_{1} \supset A_{2} \supset \cdots \supset$ $A_{n} \supset \cdots$.

Let $A$ be an open set of $X$ such that $f^{-1}(p) \subseteq A$ and suppose that $f^{-1}\left(A_{n}\right) \nsubseteq A$, for all $n \geq 1$. Then $\left\{f^{-1}\left(A_{n}\right) \backslash A\right\}_{n \geq 1}$ is a decreasing sequence of non void compact sets, hence $K=\left(\bigcap_{n \geq 1} f^{-1}\left(A_{n}\right)\right) \backslash A \neq \varnothing$. It follows that $f(K)=\{p\}$, which contradicts the hypothesis $f^{-1}(p) \subseteq A$.

Let $\sigma \in \Sigma^{(n)}$ and $J \subseteq\{1, \ldots, n\}$, then we define the subset of $\boldsymbol{K}^{n}(\sigma)$ given by $E_{\sigma, J}=$ $\left\{y_{\sigma} \in W_{\sigma}: y_{\sigma, j}=0, \forall j \in J\right\}$.

Lemma 4.4. Let $\sigma \in \Sigma^{(n)}$ and $J \subseteq\{1, \ldots, n\}$. Then, $\Delta=\bigcap_{j \in J} \Delta\left(a^{j}(\sigma), \Gamma_{+}\right)$is a compact face of $\Gamma_{+}$if and only if $\pi_{\sigma}\left(E_{\sigma, J}\right)=\{0\}$.

Proof. We observe that the condition $\pi_{\sigma}\left(E_{\sigma, J}\right)=\{0\}$ is equivalent to say that all the coordinates of the vector $\sum_{j \in J} a^{j}(\sigma)$ are positive, but this implies that the set

$$
\left\{k \in \Gamma_{+}:\left\langle k, \sum_{j \in J} a^{j}(\sigma)\right\rangle=\sum_{j \in J} \ell\left(a^{j}(\sigma), \Gamma_{+}\right)\right\} \cap \boldsymbol{Z}_{+}^{n}
$$

is finite. Therefore, the result follows from the following equality:

$$
\bigcap_{j \in J} \Delta\left(a^{j}(\sigma), \Gamma_{+}\right)=\left\{k \in \Gamma_{+}:\left\langle k, \sum_{j \in J} a^{j}(\sigma)\right\rangle=\sum_{j \in J} \ell\left(a^{j}(\sigma), \Gamma_{+}\right)\right\} .
$$

Let $\sigma \in \Sigma^{(n)}$ and let $x^{k}=x_{1}^{k_{1}} \cdots x_{n}^{k_{n}}$ be a monomial, then we observe that

$$
x^{k} \circ \pi_{\sigma}\left(y_{\sigma}\right)=y_{\sigma, 1}^{\left\langle k, a^{1}(\sigma)\right\rangle} \cdots y_{\sigma, n}^{\left\langle k, a^{n}(\sigma)\right\rangle} \text {. }
$$

Thus, given the system $S=\left\{g_{1}, \ldots, g_{s}\right\} \subseteq A\left(\boldsymbol{K}^{n}\right)$ and $\sigma \in \Sigma^{(n)}$, we can consider the associated system $S_{\sigma}=\left\{\tilde{g}_{\sigma, 1}, \ldots, \tilde{g}_{\sigma, s}\right\} \subseteq A\left(\boldsymbol{K}^{n}(\sigma)\right)$, where each $\tilde{g}_{\sigma, i}$ is defined by the equations

$$
g_{i} \circ \pi_{\sigma}\left(y_{\sigma}\right)=y_{\sigma, 1}^{\ell_{i}\left(a^{1}(\sigma)\right)} \cdots y_{\sigma, n}^{\ell_{i}\left(a^{n}(\sigma)\right)} \cdot \tilde{g}_{\sigma, i}\left(y_{\sigma}\right),
$$

and we write $\ell_{i}(v)$ for the number $\ell\left(v, g_{i}\right)$, for all $i=1, \ldots, s$, and all $v \in \boldsymbol{R}_{+}^{n} \backslash\{0\}$.

Proposition 4.5. If the system $S$ is adapted to $\Gamma_{+}$then $\sup _{i}\left|\tilde{g}_{\sigma, i}\left(y_{\sigma}\right)\right| \neq 0$, for all $\sigma \in \Sigma^{(n)}$ and all $y_{\sigma} \in \pi_{\sigma}^{-1}(0)$. 
Proof. Let us fix an index $i \in\{1, \ldots, s\}$ and a cone $\sigma \in \Sigma^{(n)}$. Given a subset $J \subseteq\{1, \ldots, n\}, J \neq \varnothing$, we define

$$
\sigma_{J}=\left\{k \in \Gamma_{+}\left(g_{i}\right):\left\langle k, a^{j}(\sigma)\right\rangle=\ell_{i}\left(a^{j}(\sigma)\right), \forall j \in J, \text { and }\left\langle k, a^{j}(\sigma)\right\rangle>\ell_{i}\left(a^{j}(\sigma)\right), \forall j \notin J\right\} .
$$

It is clear that, if $J_{1}, J_{2} \subseteq\{1, \ldots, n\}$ and $\sigma_{J_{1}} \cap \sigma_{J_{2}} \neq \varnothing$ then $J_{1}=J_{2}$. Then, if we define $Z_{i}=\left\{k \in \operatorname{supp}\left(g_{i}\right):\left\langle k, a^{j}(\sigma)\right\rangle=\ell_{i}\left(a^{j}(\sigma)\right)\right.$, for some $\left.j \in\{1, \ldots, n\}\right\}$ and we suppose that $g_{i}=\sum_{k} a_{k} x^{k}$, we can write the following:

$$
\begin{aligned}
g_{i} \circ \pi_{\sigma}\left(y_{\sigma}\right)= & \left(\sum_{k \in Z_{i}} a_{k} x^{k}+\sum_{k \notin Z_{i}} a_{k} x^{k}\right) \circ \pi_{\sigma}\left(y_{\sigma}\right) \\
= & \sum_{J \subseteq\{1, \ldots, n\}} \sum_{k \in \sigma_{J}} a_{k} y_{\sigma, 1}^{\ell_{i}\left(a^{1}(\sigma)\right)} \cdots y_{\sigma, n}^{\ell_{i}\left(a^{n}(\sigma)\right)} \prod_{j=1}^{n} y_{\sigma, j}^{\left\langle k, a^{j}(\sigma)\right\rangle-\ell_{i}\left(a^{j}(\sigma)\right)} \\
& +\sum_{k \notin Z_{i}} a_{k} x^{k} \circ \pi_{\sigma}\left(y_{\sigma}\right) \\
= & y_{\sigma, 1}^{\ell_{i}\left(a^{1}(\sigma)\right)} \cdots y_{\sigma, n}^{\ell_{i}\left(a^{n}(\sigma)\right)}\left(\sum_{J \subseteq\{1, \ldots, n\}} \sum_{k \in \sigma_{J}} a_{k} \prod_{j \notin J} y_{\sigma, j}^{\left\langle k, a^{j}(\sigma)\right\rangle-\ell_{i}\left(a^{j}(\sigma)\right)}\right. \\
& \left.+\sum_{k \notin Z_{i}} a_{k} \prod_{j=1}^{n} y_{\sigma, j}^{\left\langle k, a^{j}(\sigma)\right\rangle-\ell_{i}\left(a^{j}(\sigma)\right)}\right) .
\end{aligned}
$$

Then, we observe that the germ $\tilde{g}_{\sigma, i}$ defined in (2) is equal to the expression in parenthesis of the last equality.

Let $y_{\sigma} \in \pi_{\sigma}^{-1}(0)$ such that $\tilde{g}_{\sigma, i}\left(y_{\sigma}\right)=0$, for all $i=1, \ldots, s$, and let $H=\left\{j: y_{\sigma, j}=0\right\}$. Since $y_{\sigma} \in \pi_{\sigma}^{-1}(0)$, the set $H$ is non empty. This implies that

$$
\tilde{g}_{\sigma, i}\left(y_{\sigma}\right)=\sum_{H \subseteq J \subseteq\{1, \ldots, n\}} \sum_{k \in \sigma_{J}} a_{k} \prod_{j \notin J} y_{\sigma, j}^{\left\langle k, a^{j}(\sigma)\right\rangle-\ell_{i}\left(a^{j}(\sigma)\right)}
$$

The closure $\bar{\sigma}_{H}$ of $\sigma_{H}$ in $\boldsymbol{R}_{+}^{n}$ is expressed as

$$
\begin{aligned}
\bar{\sigma}_{H} & =\left\{k \in \Gamma_{+}\left(g_{i}\right):\left\langle k, a^{j}(\sigma)\right\rangle=\ell_{i}\left(a^{j}(\sigma)\right), \forall j \in H \text { and }\left\langle k, a^{j}(\sigma)\right\rangle \geq \ell_{i}\left(a^{j}(\sigma)\right), \forall j \notin H\right\} \\
& =\Delta\left(H^{\prime}, g_{i}\right)
\end{aligned}
$$

where $H^{\prime} \subseteq\{1, \ldots, r\}$ is the set of indexes $i(j)$ such that $a^{j}(\sigma)=v^{i(j)}$, for some $j \in H$. Let $\left(g_{i}\right)_{\bar{\sigma}_{H}}$ the sum of those $a_{k} x^{k}$ such that $k \in \bar{\sigma}_{H}$, or equivalently, let $\left(g_{i}\right)_{\bar{\sigma}_{H}}=q_{H^{\prime}}\left(g_{i}\right)$. Therefore, for any $z_{\sigma}=\left(z_{\sigma, 1}, \ldots, z_{\sigma, n}\right) \in \boldsymbol{K}^{n}(\sigma)$ we have the following expression:

$$
\left(g_{i}\right)_{\bar{\sigma}_{H}} \circ \pi_{\sigma}\left(z_{\sigma}\right)=z_{\sigma, 1}^{\ell_{i}\left(a^{1}(\sigma)\right)} \cdots z_{\sigma, n}^{\ell_{i}\left(a^{n}(\sigma)\right)}\left(\sum_{H \subseteq J \subseteq\{1, \ldots, n\}} \sum_{k \in \sigma_{J}} a_{k} \prod_{j \notin J} z_{\sigma, j}^{\left\langle k, a^{j}(\sigma)\right\rangle-\ell_{i}\left(a^{j}(\sigma)\right)}\right) .
$$

In particular, if we consider the point $\tilde{y}_{\sigma} \in \boldsymbol{K}^{n}(\sigma)$ given by $\tilde{y}_{\sigma, j}=y_{\sigma, j}$, when $j \notin H$ and $\tilde{y}_{\sigma, j}=1$, when $j \in H$, we conclude that 


$$
\begin{aligned}
\left(g_{i}\right)_{\bar{\sigma}_{H}} \circ \pi_{\sigma}\left(\tilde{y}_{\sigma}\right) & =\tilde{y}_{\sigma, 1}^{\ell_{i}\left(a^{1}(\sigma)\right)} \cdots \tilde{y}_{\sigma, n}^{\ell_{i}\left(a^{n}(\sigma)\right)}\left(\sum_{H \subseteq J \subseteq\{1, \ldots, n\}} \sum_{k \in \sigma_{J}} a_{k} \prod_{j \notin J} y_{\sigma, j}^{\left\langle k, a^{j}(\sigma)\right\rangle-\ell_{i}\left(a^{j}(\sigma)\right)}\right) \\
& =\tilde{y}_{\sigma, 1}^{\ell_{i}\left(a^{1}(\sigma)\right)} \cdots \tilde{y}_{\sigma, n}^{\ell_{i}\left(a^{n}(\sigma)\right)} \cdot \tilde{g}_{\sigma, i}\left(y_{\sigma}\right)=0,
\end{aligned}
$$

where the second equality follows from (3).

Since $\pi_{\sigma}\left(E_{\sigma, H}\right)=\{0\}$, the set $\Delta=\bigcap_{j \in H} \Delta\left(a^{j}(\sigma), \Gamma_{+}\right)=\bigcap_{j \in H} \Delta\left(v^{i(j)}, \Gamma_{+}\right)$is a compact face of $\Gamma_{+}$, by Lemma 4.4. On the other hand, we have that $q_{H^{\prime}}\left(g_{i}\right) \circ \pi_{\sigma}\left(\tilde{y}_{\sigma}\right)=$ $\left(g_{i}\right)_{\bar{\sigma}_{H}} \circ \pi_{\sigma}\left(\tilde{y}_{\sigma}\right)=0$, for all $i=1, \ldots, s$, which contradicts the hypothesis that $S$ is an adapted system to $\Gamma_{+}$, since $\pi_{\sigma}\left(\tilde{y}_{\sigma}\right) \in(\boldsymbol{K} \backslash\{0\})^{n}$.

Proof of Theorem 3.5. From the hypotheses of the theorem and the fact that the set of 1-dimensional cones of $\Sigma_{0}$ is equal to the set of 1-dimensional cones of $\Sigma$ we have that

$$
\left\langle k, a^{j}(\sigma)\right\rangle \geq \theta \max \left\{\ell_{1}\left(a^{j}(\sigma)\right), \ldots, \ell_{s}\left(a^{j}(\sigma)\right)\right\},
$$

for all $j=1, \ldots, n$ and all $\sigma \in \Sigma^{(n)}$. Given any cone $\sigma \in \Sigma^{(n)}$, this implies the inequality (4) $\left|x^{k}\right|^{1 / \theta} \circ \pi_{\sigma}\left(y_{\sigma}\right)$

$$
=\left|y_{\sigma, 1}\right|^{\left\langle k, a^{1}(\sigma)\right\rangle / \theta} \cdots\left|y_{\sigma, n}\right|^{\left\langle k, a^{n}(\sigma)\right\rangle / \theta} \leq\left|y_{\sigma, 1}\right|^{\max _{i} \ell_{i}\left(a^{1}(\sigma)\right)} \cdots\left|y_{\sigma, n}\right|^{\max _{i} \ell_{i}\left(a^{n}(\sigma)\right)},
$$

for every $y_{\sigma} \in W_{\sigma}$.

Let $U$ be a neighbourhood of $0 \in \boldsymbol{K}^{n}$ where all the functions $g_{i}$ are defined. Then, we have the following inequalities

$$
\begin{aligned}
\sup _{i}\left|g_{i} \circ \pi_{\sigma}\left(y_{\sigma}\right)\right| & =\sup _{i}\left|y_{\sigma, 1}^{\ell_{i}\left(a^{1}(\sigma)\right)} \cdots y_{\sigma, n}^{\ell_{i}\left(a^{n}(\sigma)\right)} \cdot \tilde{g}_{\sigma, i}\left(y_{\sigma}\right)\right| \\
& \geq\left|y_{\sigma, 1}\right|^{\max _{i} \ell_{i}\left(a^{1}(\sigma)\right)} \cdots\left|y_{\sigma, n}\right|^{\max _{i} \ell_{i}\left(a^{n}(\sigma)\right)} \sup _{i}\left|\tilde{g}_{\sigma, i}\left(y_{\sigma}\right)\right|,
\end{aligned}
$$

for all $y_{\sigma} \in \pi_{\sigma}^{-1}(U) \subseteq W_{\sigma}$.

Since $S$ is an adapted system to $\Gamma_{+}$, it follows that $\sup _{i}\left|\tilde{g}_{\sigma, i}\left(y_{\sigma}\right)\right| \neq 0$, for all $y_{\sigma} \in \pi_{\sigma}^{-1}(0)$, by Proposition 4.5. This implies that, for any $\sigma \in \Sigma^{(n)}$, we can find a compact neighbourhood $A_{\sigma}$ of $\pi_{\sigma}^{-1}(0)$ contained in $W_{\sigma}$ such that $\sup _{i}\left|\tilde{g}_{\sigma, i}\left(y_{\sigma}\right)\right| \neq 0$, for all $y_{\sigma} \in A_{\sigma}$. Then, the number $c_{\sigma}=\inf _{y_{\sigma} \in A_{\sigma}} \sup _{i}\left|\tilde{g}_{\sigma, i}\left(y_{\sigma}\right)\right|$ is a positive constant.

Joining the information above, we get that

$$
\begin{aligned}
\sup _{i}\left|g_{i} \circ \pi_{\sigma}\left(y_{\sigma}\right)\right| & \geq\left|y_{\sigma, 1}\right|^{\max _{i} \ell_{i}\left(a^{1}(\sigma)\right)} \cdots\left|y_{\sigma, n}\right|^{\max _{i} \ell_{i}\left(a^{n}(\sigma)\right)} \sup _{i}\left|\tilde{g}_{\sigma, i}\left(y_{\sigma}\right)\right| \\
& \geq c_{\sigma}\left|y_{\sigma, 1}\right|^{\max _{i} \ell_{i}\left(a^{1}(\sigma)\right)} \cdots\left|y_{\sigma, n}\right|^{\max _{i} \ell_{i}\left(a^{n}(\sigma)\right)} \\
& \geq c_{\sigma}\left|x^{k}\right|^{1 / \theta} \circ \pi_{\sigma}\left(y_{\sigma}\right),
\end{aligned}
$$

for all $y_{\sigma} \in A_{\sigma}$.

Let $c=\min _{\sigma} c_{\sigma}$, where $\sigma$ varies in $\Sigma^{(n)}$. Then, we have that

$$
\sup _{i}\left|g_{i} \circ \pi_{\sigma}\left(y_{\sigma}\right)\right| \geq c\left|x^{k}\right|^{1 / \theta} \circ \pi_{\sigma}\left(y_{\sigma}\right),
$$

for all $y_{\sigma} \in A_{\sigma}$. If $C=1 / c$, we now conclude that 


$$
\left|x^{k}\right|^{1 / \theta} \leq C \sup _{i}\left|g_{i}(x)\right|
$$

for all $x$ in an open neighbourhood of 0 in $\boldsymbol{K}^{n}$, by Lemmas 4.2 and 4.3. But this means that $x^{k} \in \overline{I^{\theta}}$, by Definition 2.1, and hence Theorem 3.5 is proved.

If we know explicitly the partition $\Sigma$ constructed in Section 4 we can improve the statement of Theorem 3.5 as follows. Suppose that $S=\left\{g_{1}, \ldots, g_{s}\right\} \subseteq A\left(\boldsymbol{K}^{n}\right)$ is an adapted system to $\Gamma_{+}$. If $\sigma \in \Sigma^{(n)}$, let $H_{\sigma} \subseteq\{1, \ldots, r\}$, the set of indexes $i(j)$ such that $v^{i(j)}=a^{j}(\sigma)$, for some $j \in\{1, \ldots, n\}$. Then, for each $\sigma \in \Sigma^{(n)}$, we can choose a subset $A_{\sigma} \subseteq\{1, \ldots, s\}$ with the condition that $\left\{x \in \boldsymbol{K}^{n}: q_{H^{\prime}}\left(g_{i}\right)(x)=0, \forall i \in A_{\sigma}\right\} \subseteq$ $\left\{x \in K^{n}: x_{1} \cdots x_{n}=0\right\}$, for all $H^{\prime} \subseteq H_{\sigma}$ such that $\bigcap_{j \in H^{\prime}} \Delta\left(v^{j}, \Gamma_{+}\right)$is a compact face of $\Gamma_{+}$. Then, we obtain the following result, which is particularly useful when $n=2$ (see Remark 4.1).

THEOREM 4.6. In the conditions described above, let $I \subseteq A\left(\boldsymbol{K}^{n}\right)$ be the ideal generated by $S$. Let $x^{k}$ be any monomial such that $\left\langle k, a^{j}(\sigma)\right\rangle \geq \theta \max _{i \in A_{\sigma}} \ell\left(a^{j}(\sigma), g_{i}\right)$, for every $\sigma \in \Sigma^{(n)}$ and every $j=1, \ldots, n$. Then $x^{k} \in \overline{I^{\theta}}$.

ProOF. It is a direct consequence of the proof arguments of Proposition 4.5 and Theorem 3.5.

\section{Strongly adapted systems.}

In this section, we show a special kind of adapted systems to Newton polyhedrons. If $S \subseteq A\left(\boldsymbol{K}^{n}\right)$ is one of these systems and $I$ is the ideal of $A\left(\boldsymbol{K}^{n}\right)$ generated by $S$, we will obtain a monomial $x^{k}$ belonging to $\overline{I^{\theta}}$ whose exponent $k$ belongs to any fixed coordinate axis, where $\theta$ is a given rational number. This is not always possible if we apply Theorem 3.5 to an arbitrary adapted system to a given Newton polyhedron, since $\max _{i} \ell\left(v^{j}, g_{i}\right)$ could be positive when $v^{j}$ is some vector of the canonical basis in $\boldsymbol{R}^{n}$.

Throughout this section, we will assume that $\Gamma_{+} \subseteq \boldsymbol{R}^{n}$ is a Newton polyhedron intersecting all coordinate axis. As is easy to check, in this situation we will have that $\left\{v \in \boldsymbol{R}_{+}^{n} \backslash\{0\}: \Delta(v)\right.$ is not compact $\}=\left\{v \in \boldsymbol{R}_{+}^{n} \backslash\{0\}: \ell\left(v, \Gamma_{+}\right)=0\right\}$.

Hence, if $v^{1}, \ldots, v^{r}$ are the primitive vectors supporting some $(n-1)$-dimensional face of $\Gamma_{+}$, we can suppose that $v^{1}, \ldots, v^{\ell}$ support some compact face of $\Gamma_{+}$, where $\ell<r$ and $n=r-\ell$, and that the least $n$ vectors form the canonical basis in $\boldsymbol{R}^{n}$.

Definition 5.1. If $g \in A\left(\boldsymbol{K}^{n}\right)$ and $j \in\{1, \ldots, r\}$, we define $\ell^{*}\left(v^{j}, g\right)=\ell\left(v^{j}, g\right)$, when $\Delta\left(v^{j}, \Gamma_{+}\right)$is a compact face of $\Gamma_{+}$, and $\ell^{*}\left(v^{j}, g\right)=0$, otherwise. Therefore, we also define $\Delta^{*}(J, g)=\left\{k \in \operatorname{supp}(g):\left\langle k, v^{j}\right\rangle=\ell^{*}\left(v^{j}, g\right), \forall j \in J\right\}$.

Given $g=\sum_{k} a_{k} x^{k} \in A\left(\boldsymbol{K}^{n}\right)$ and $J \subseteq\{1, \ldots, r\}$, we denote by $q_{J}^{*}(g)$ the polynomial obtained as the sum of those $a_{k} x^{k}$ such that $k \in \Delta^{*}(J, g)$. If $\Delta^{*}(J, g)=\varnothing$, then we shall fix $q_{J}^{*}(g)=0$.

Definition 5.2. We say that $S=\left\{g_{1}, \ldots, g_{s}\right\} \subseteq A\left(\boldsymbol{K}^{n}\right)$ is a strongly adapted system to $\Gamma_{+}$when, for all $J \subseteq\{1, \ldots, r\}$ such that $\bigcap_{j \in J} \Delta\left(v^{j}, \Gamma_{+}\right)$is a compact face of $\Gamma_{+}$, the system of equations

$$
q_{J}^{*}\left(g_{1}\right)(x)=\cdots=q_{J}^{*}\left(g_{s}\right)(x)=0
$$


has no solution in $(\boldsymbol{K} \backslash\{0\})^{n}$. Moreover, we say that $S$ is a strongly adapted system when $S$ is strongly adapted to the Newton polyhedron determined by $S$.

If $g \in A\left(\boldsymbol{K}^{n}\right)$ and $J \subseteq\{1, \ldots, r\}$ is such that $\Delta=\bigcap_{j \in J} \Delta\left(v^{j}, \Gamma_{+}\right)$is a compact face of $\Gamma_{+}$, then $q_{J}^{*}(g)=q_{J}(g)$ or $q_{J}^{*}(g)=0$. The equality $q_{J}^{*}(g)=0$ holds when there exists some $j \in J$ such that $\Delta\left(v^{j}, \Gamma_{+}\right)$is not a compact face of $\Gamma_{+}$and $\ell\left(v^{j}, g\right)>0$. Then, we see that every strongly adapted system to $\Gamma_{+}$is adapted to $\Gamma_{+}$.

If $L \subseteq\{1, \ldots, n\}$, we define $\left(\boldsymbol{R}^{n}\right)_{L}=\left\{x \in \boldsymbol{R}^{n}: x_{j}=0, \forall j \in L\right\}$ and $\left(\Gamma_{+}\right)_{L}=$ $\Gamma_{+} \cap\left(\boldsymbol{R}^{n}\right)_{L}$ when $\Gamma_{+} \subseteq \boldsymbol{R}^{n}$ is a Newton polyhedron. If $g=\sum_{k} a_{k} x^{k} \in A\left(\boldsymbol{K}^{n}\right)$, then we denote by $g_{L}$ the polynomial obtained as the sum of those $a_{k} x^{k}$ such that $k \in \operatorname{supp}(g) \cap\left(\boldsymbol{R}^{n}\right)_{L}$. If $S=\left\{g_{1}, \ldots, g_{s}\right\} \subseteq A\left(\boldsymbol{K}^{n}\right)$, we write $S_{L}$ to denote the system $\left\{g_{1_{L}}, \ldots, g_{s_{L}}\right\}$.

For each $J \subseteq\{1, \ldots, r\}, J \neq \varnothing$, we set $J^{*}=\left\{i \in\{1, \ldots, n\}: e_{i}\right.$ is equal to some vector $\left.v^{j}, j \in J\right\}$. If we define $J_{1}=J \cap\{1, \ldots, \ell\}$ and $J_{2}=J \cap\{\ell+1, \ldots, r\}$, it is straightforward to see that $q_{J}^{*}(g)=q_{J_{1}}\left(g_{J_{2}^{*}}\right)$, for all $g \in A\left(\boldsymbol{K}^{n}\right)$. In particular, we observe that $S$ is a strongly adapted system to $\Gamma_{+}$if and only if $S_{L}$ is an adapted system to $\left(\Gamma_{+}\right)_{L}$, for all $L \subseteq\{1, \ldots, n\}$

EXAMPLE 5.3. Let us consider the system $S=\left\{x^{5}+x y(x-y)^{2}, y^{5}+x y(x-y)^{2}\right.$, $\left.z^{3}\right\} \subseteq A\left(\boldsymbol{R}^{3}\right)$. This system is adapted, since it contains a monomial, but it is not strongly adapted, because the system $S^{\prime}=\left\{x^{5}+x y(x-y)^{2}, y^{5}+x y(x-y)^{2}\right\}$ is not adapted.

Now we state an analogous result to Theorem 3.5 for strongly adapted systems. In this case, we shall impose conditions depending on the compact faces of $\Gamma_{+}$to a given $k \in \boldsymbol{R}^{n}$ in order to obtain that $x^{k} \in \overline{I^{\theta}}$, where $\theta \in \boldsymbol{Q}_{+}$.

COROLlary 5.4. Let $S=\left\{g_{1}, \ldots, g_{s}\right\} \subseteq A\left(\boldsymbol{K}^{n}\right)$ be a strongly adapted system to $\Gamma_{+}$ such that $V(S)=\{0\}$. Let $I \subseteq A\left(\boldsymbol{K}^{n}\right)$ be the ideal generated by $S$ and let $\theta \in \boldsymbol{Q}_{+}$. If $x^{k}$ is a monomial such that $\left\langle k, v^{j}\right\rangle \geq \theta \max \left\{\ell\left(v^{j}, g_{1}\right), \ldots, \ell\left(v^{j}, g_{s}\right)\right\}$, for all $j=1, \ldots, \ell$, then $x^{k} \in \overline{I^{\theta}}$.

Proof. Since $V(I)=\{0\}$, there exists some $N \geq 1$ such that $m_{n}^{N} \subseteq \bar{I}$, by [12, p. 136] (see Definition 2.1). Here we denote by $m_{n}$ the maximal ideal of $A\left(\boldsymbol{K}^{n}\right)$. Let $G(x)=x_{1}^{N}+\cdots+x_{n}^{N}$ and consider the system given by $S^{\prime}=\left\{g_{1}+G, \ldots, g_{n}+G\right\}$. Then we have the inclusion

$$
\overline{\left\langle g_{1}+G, \ldots, g_{s}+G\right\rangle^{\theta}} \subseteq \overline{I^{\theta}},
$$

for any $\theta \in \boldsymbol{Q}_{+}$.

If $j \in\{1, \ldots, \ell\}$, suppose that $v^{j}=\left(v_{1}^{j}, \ldots, v_{n}^{j}\right)$ and let $v_{0}^{j}=\min \left\{v_{1}^{j}, \ldots, v_{n}^{j}\right\}$. Given any $i \in\{1, \ldots, s\}$, we have that

$$
\begin{aligned}
\ell\left(v^{j}, g_{i}+G\right)=\ell\left(v^{j}, g_{i}\right) & \Leftrightarrow \ell\left(v^{j}, g_{i}\right) \leq \min \left\{\left\langle k, v^{j}\right\rangle: k \in \operatorname{supp}(G)\right\} \\
& \Leftrightarrow \ell\left(v^{j}, g_{i}\right) \leq \min \left\{N v_{t}^{j}: t=1, \ldots, n\right\} \\
& \Leftrightarrow N \geq \frac{\ell\left(v^{j}, g_{i}\right)}{v_{0}^{j}} .
\end{aligned}
$$


Therefore, if $N$ satisfies the inequality

$$
N \geq \max _{i, j} \frac{\ell\left(v^{j}, g_{i}\right)}{v_{0}^{j}},
$$

where $j \in\{1, \ldots, \ell\}$ and $i \in\{1, \ldots, s\}$, then $\ell\left(v^{j}, g_{i}+G\right)=\ell\left(v^{j}, g_{i}\right)$, for all $j \in\{1, \ldots, \ell\}$.

Moreover, by the definition of $G$, we have that $\ell\left(v^{j}, g_{i}+G\right)=0$, for all $j=$ $\ell+1, \ldots, r$. Hence, we get the equality $q_{J}^{*}\left(g_{i}\right)=q_{J}\left(g_{i}+G\right)$, for all $i=1, \ldots, s$ and all $J \subseteq\{1, \ldots, r\}$ such that $\Delta=\bigcap_{j \in J} \Delta\left(v^{j}, \Gamma_{+}\right)$is a compact face of $\Gamma_{+}$. But this implies that $S^{\prime}$ is an adapted system to $\Gamma_{+}$, since $S$ is strongly adapted to $\Gamma_{+}$. Then, applying Theorem 3.5 to the system $S^{\prime}$ and considering the inclusion (6), we obtain the desired result.

In the conditions of the above corollary, we define the following subset of $\boldsymbol{R}_{+}^{n}$ :

$$
M_{\theta}\left(S, \Gamma_{+}\right)=\left\{k \in \boldsymbol{R}_{+}^{n}:\left\langle k, v^{j}\right\rangle \geq \theta \max \left\{\ell\left(v^{j}, g_{1}\right), \ldots, \ell\left(v^{j}, g_{s}\right)\right\}, \forall j=1, \ldots, \ell\right\} .
$$

We observe that the set $M_{\theta}\left(S, \Gamma_{+}\right)$intersects each coordinate axis. When $\theta=1$, we denote the set $M_{\theta}\left(S, \Gamma_{+}\right)$simply by $M\left(S, \Gamma_{+}\right)$.

\section{Estimation of Lojasiewicz exponents.}

In this section, we apply Corollary 5.4 to obtain a result estimating Łojasiewicz exponents. Along this section we will suppose that $\Gamma_{+} \subseteq \boldsymbol{R}^{n}$ is a Newton polyhedron intersecting all coordinate axis. As in the previous section, we denote by $v^{1}, \ldots, v^{\ell}$ the primitive vectors supporting some compact face of dimension $n-1$ of $\Gamma_{+}$.

THEOREM 6.1. Let $S=\left\{g_{1}, \ldots, g_{s}\right\} \subseteq A\left(\boldsymbol{K}^{n}\right)$ be a strongly adapted system to $\Gamma_{+}$ such that $V(S)=\{0\}$ and let $I \subseteq A\left(\boldsymbol{K}^{n}\right)$ be the ideal generated by $S$. Let $J=$ $\left\langle h_{1}, \ldots, h_{t}\right\rangle \subseteq A\left(\boldsymbol{K}^{n}\right)$ and suppose that $0 \in V(J)$, then

$$
\ell(J, I) \leq \max _{j=1, \ldots, \ell} \frac{\max \left\{\ell\left(v^{j}, g_{1}\right), \ldots, \ell\left(v^{j}, g_{s}\right)\right\}}{\min \left\{\ell\left(v^{j}, h_{1}\right), \ldots, \ell\left(v^{j}, h_{t}\right)\right\}}
$$

Proof. Considering Definition 2.1 and Corollary 5.4, we obtain the following inequalities:

$$
\begin{aligned}
\ell(J, I) & =\inf \left\{\theta \in \boldsymbol{Q}: J \subseteq \overline{I^{1 / \theta}}\right\} \\
& \leq \inf \left\{\theta \in \boldsymbol{Q}: \Gamma_{+}(J) \subseteq M_{1 / \theta}\left(S, \Gamma_{+}\right)\right\} \\
& =\inf \left\{\theta \in \boldsymbol{Q}: \ell\left(v^{j}, h_{i}\right) \geq \frac{1}{\theta} \max \left\{\ell\left(v^{j}, g_{1}\right), \ldots, \ell\left(v^{j}, g_{s}\right)\right\}, \text { for all } i, j\right\} \\
& =\max _{j=1, \ldots, \ell} \frac{\max \left\{\ell\left(v^{j}, g_{1}\right), \ldots, \ell\left(v^{j}, g_{s}\right)\right\}}{\min \left\{\ell\left(v^{j}, h_{1}\right), \ldots, \ell\left(v^{j}, h_{t}\right)\right\}} .
\end{aligned}
$$

If we replace the ideal $J$ by the maximal ideal in Theorem 6.1, the following upper bound for the Lojasiewicz exponent $\ell\left(m_{n}, I\right)$ is obtained: 


$$
\ell\left(m_{n}, I\right) \leq \max _{j=1, \ldots, \ell}\left\{\frac{\ell\left(v^{j}, g_{1}\right)}{v_{0}^{j}}, \ldots, \frac{\ell\left(v^{j}, g_{s}\right)}{v_{0}^{j}}\right\}
$$

where $v_{0}=\min \left\{v_{1}, \ldots, v_{n}\right\}$, for any $v=\left(v_{1}, \ldots, v_{n}\right) \in(\boldsymbol{R} \backslash\{0\})^{n}$.

If $f:\left(\boldsymbol{K}^{n}, 0\right) \rightarrow(\boldsymbol{K}, 0)$ is an analytic function germ with an isolated singularity at the origin, we denote by $\alpha_{0}(f)$ the Lojasiewicz exponent $\ell\left(m_{n}, J(f)\right)$, where $J(f)$ is the jacobian ideal of $f$. We shall denote by $S_{f}$ the system of $A\left(\boldsymbol{K}^{n}\right)$ formed by the partial derivatives of $f$ and we call this the jacobian system of $f$.

If $v=\left(v_{1}, \ldots, v_{n}\right) \in(\boldsymbol{R} \backslash\{0\})^{n}$ and $a \geq 0$, then the largest of the distances from the origin to some point in the intersection of the union of the coordinate axis with the plane $\langle k, v\rangle=a$ is equal to $a / v_{0}$. From this observation, together with Remark 2.2, we obtain the following consequence of Theorem 6.1.

Corollary 6.2. Let $f:\left(\boldsymbol{K}^{n}, 0\right) \rightarrow(\boldsymbol{K}, 0)$ be an analytic function germ with an isolated singularity at the origin, let $S=\left\{g_{1}, \ldots, g_{s}\right\} \subseteq A\left(\boldsymbol{K}^{n}\right)$ and let $I$ be the ideal generated by $S$. Suppose that $S$ is strongly adapted to $\Gamma_{+}$and that $\bar{I}=\overline{J(f)}$. Let $P_{1}, \ldots, P_{n}$ the points in the intersection of $M\left(S, \Gamma_{+}\right)$and the union of the coordinate axis and let $r=\max \left\{\left|P_{1}\right|, \ldots,\left|P_{n}\right|\right\}$. Then $\alpha_{0}(f) \leq r$.

For the sake of completeness, we state the result of Fukui [5] on the estimation of the Lojasiewicz exponent $\alpha_{0}(f)$ of an analytic function germ. First, we need to give some preliminary definitions.

Suppose that $u^{1}, \ldots, u^{m}$ is the family of primitive vectors supporting some compact face of dimension $n-1$ of the Newton polyhedron $\Gamma_{+}(f)$ of $f$. Then, we denote by $m_{0}(f)$ the largest of the distances from the origin to some point in the intersection of the union of the coordinate axis with some plane of equation $\left\langle k, u^{j}\right\rangle=\ell\left(u^{j}, f\right)$, where $j=1, \ldots, m$.

Given a series $g=\sum_{k} a_{k} x^{k} \in A\left(\boldsymbol{K}^{n}\right)$ and a compact subset $\Delta \subseteq \boldsymbol{R}^{n}$, we denote by $g_{\Delta}$ the polynomial obtained by the sum of those $a_{k} x^{k}$ such that $k \in \Delta$. We recall that an analytic function germ $f:\left(\boldsymbol{K}^{n}, 0\right) \rightarrow(\boldsymbol{K}, 0)$ is said to be Newton non-degenerate when, for each compact face $\Delta \subseteq \Gamma_{+}(f)$, the system of equations $\left(x_{1} \partial f / \partial x_{1}\right)_{\Delta}=\cdots=$ $\left(x_{n} \partial f / \partial x_{n}\right)_{\Delta}=0$ has no solution in $(\boldsymbol{K} \backslash\{0\})^{n}$ (see [8] and [17] for more information on this kind of functions). If $f$ is not Newton non-degenerate, then we say that $f$ is Newton degenerate.

THEOREM $6.3([\mathbf{5}])$. Let $f:\left(\boldsymbol{K}^{n}, 0\right) \rightarrow(\boldsymbol{K}, 0)$ be an analytic map germ with an isolated singularity at the origin. Suppose that $f$ is Newton non-degenerate, then $\alpha_{0}(f) \leq$ $m_{0}(f)-1$.

The above theorem was proved by Lichtin [11] in the case $n=2$ under stronger conditions.

REMARK 6.4. i) As we see, Theorem 6.3 uses the Newton polyhedron of the function $f$ as a tool to obtain an upper estimation for $\alpha_{0}(f)$. On the other hand, in Corollary 6.2 we are considering a Newton polyhedron $\Gamma_{+}$such that $\overline{J(f)}$ is determined by a strongly adapted system $S$ to $\Gamma_{+}$. Observe that $S$ must not be equal to $S_{f}$.

ii) The next example shows that the class of those functions whose jacobian system 
$S_{f}$ is strongly adapted to some Newton polyhedron is not included in the class of Newton non-degenerate function germs.

EXAmple 6.5. Let $f:\left(\boldsymbol{R}^{3}, 0\right) \rightarrow(\boldsymbol{R}, 0)$ given by $f(x, y, z)=(x+y)^{3}+2 x^{2} z+z^{11}$. This function is Newton degenerate and the jacobian system $S_{f}$ is strongly adapted (to its Newton polyhedron). Therefore, we have that $\alpha_{0}(f) \leq 10$, by Corollary 6.2 .

We give some examples showing how Corollary 6.2 can be applied to estimate the number $\alpha_{0}(f)$ when $f$ is Newton degenerate and the system of partial derivatives of $f$ is not strongly adapted. The following lemma is very useful in this task. We shall denote by $I(f)$ the ideal of $A\left(\boldsymbol{K}^{n}\right)$ generated by $x_{1} \partial f / \partial x_{1}, \ldots, x_{n} \partial f / \partial x_{n}$.

Lemma $6.6([\mathbf{1 6}])$. Let $f:\left(\boldsymbol{K}^{n}, 0\right) \rightarrow(\boldsymbol{K}, 0)$ be an analytic function germ. Then $f \in \overline{I(f)}$.

Proof. If $g \in A(\boldsymbol{K})$, we denote by $v(g)$ the order of $g$ at the origin. Then, by Proposition 2.3, it is enough to see that $v(f \circ \varphi) \geq \min _{i} v\left(\left(x_{i} \partial f / \partial x_{i}\right) \circ \varphi\right)$, for any analytic map germ $\varphi:(\boldsymbol{K}, 0) \rightarrow\left(\boldsymbol{K}^{n}, 0\right)$. Let $\varphi_{i}, i=1, \ldots, n$, denote the component functions of $\varphi$. If we apply the chain rule to $(d / d t)(f \circ \varphi)$, we obtain that

$$
v(f \circ \varphi)-1 \geq \min _{i}\left(v\left(\left(\partial f / \partial x_{i}\right) \circ \varphi\right)+v\left(\varphi_{i}\right)-1\right)=\min _{i} v\left(\left(x_{i} \partial f / \partial x_{i}\right) \circ \varphi-1\right),
$$

which gives the desired inequality.

EXAmPle 6.7. Consider the map germ $f:\left(\boldsymbol{R}^{3}, 0\right) \rightarrow(\boldsymbol{R}, 0)$ given by $f(x, y, z)=$ $x^{a}+y^{b}+z^{c}+\left(x^{2} y-x y^{2}\right)^{2}$, where $6 \leq a, b$ and $c>0$. It is straightforward to check that $f$ has an isolated singularity at the origin, that $f$ is Newton degenerate and that the jacobian system $S_{f}$ is not strongly adapted. On the other hand, we have that

$$
h(x, y, z):=x \frac{\partial f}{\partial x}+y \frac{\partial f}{\partial y}-6 f(x, y, z)=(a-6) x^{a}+(b-6) y^{b}-6 z^{c} \in \overline{I(f)},
$$

by Lemma 6.6. In particular, $h$ is integral over $J(f)$ and then we can apply Theorem 6.1 to the system $S=\{h, \partial f / \partial x, \partial f / \partial y, \partial f / \partial z\}$, since $S$ is strongly adapted. Therefore, we obtain that $\alpha_{0}(f) \leq \max \{a, b, c\}$.

EXAmPle 6.8. Let $f:\left(\boldsymbol{R}^{2}, 0\right) \rightarrow(\boldsymbol{R}, 0)$ given by $f(x, y)=\left(x^{2}-y^{3}\right)^{2}+y^{a}$, where $a \geq 7$. This function germ is Newton degenerate and the system $S_{f}$ is not strongly adapted. Nevertheless, by Lemma 6.6 and the relation

$$
3 x \frac{\partial f}{\partial x}+2 y \frac{\partial f}{\partial y}-12 f(x, y)=2(a-6) y^{a}
$$

we have that $y^{a} \in \overline{J(f)}$. Since the system $\left\{y^{a}, \partial f / \partial x, \partial f / \partial y\right\}$ is strongly adapted we can apply Theorem 6.1 to state that $\alpha_{0}(f) \leq a$.

Finally, we state a known result showing the relevance of the number $\alpha_{0}(f)$. For $f \in A\left(\boldsymbol{K}^{n}\right)$, we say that the $r$-jet $j^{r} f(0)$ of $f$ at $0 \in \boldsymbol{K}^{n}$ is $C^{0}$-sufficient if, for any $g \in A\left(\boldsymbol{K}^{n}\right)$ such that $j^{r} g(0)=j^{r} f(0)$, there is a germ of homeomorphism $\phi:\left(\boldsymbol{K}^{n}, 0\right) \rightarrow$ $\left(\boldsymbol{K}^{n}, 0\right)$ such that $g=f \circ \phi$. 
THEOREM 6.9 ([3], [9]). Let $f:\left(\boldsymbol{K}^{n}, 0\right) \rightarrow(\boldsymbol{K}, 0)$ be an analytic function germ with an isolated singularity at the origin, then $j^{r} f(0)$ is $C^{0}$-sufficient if $\alpha_{0}(f)<r$.

AcKnowledgement. The author wishes to express his gratitude to Professor T. Fukui for his helpful comments.

\section{References}

[1] C. Bivià-Ausina, T. Fukui and M. J. Saia, Newton graded algebras and the codimension of Newton non-degenerate ideals, Math. Proc. Cambridge Philos. Soc., 133 (2002), 55-75.

[2] J. Bochnak and J. J. Risler, Sur les exposants de Łojasiewicz, Comment. Math. Helv., 50 (1975), 493-507.

[ 3 ] S. H. Chang and Y. C. Lu, On $C^{0}$-sufficiency of complex jets, Canad. J. Math., 25 (1973), 874-880.

[4] D. Eisenbud, Commutative algebra with a view toward algebraic geometry, Grad. Texts in Math., 150, Springer-Verlag, 1994.

[5] T. Fukui, Łojasiewicz type inequalities and Newton diagrams, Proc. of the Amer. Math. Soc., 114 (1991), no. 4, 1169-1183.

[6] T. Gaffney, Integral closure of modules and Whitney equisingularity, Invent. Math., 107 (1992), 301-322.

[7] J. Gwoździewicz, The Łojasiewicz exponent of an analytic function at an isolated zero, Comment. Math. Helv., 74 (1999), 364-375.

[8] A. G. Kouchnirenko, Polyèdres de Newton et nombres de Milnor, Invent. Math., 32 (1976), 1-31.

[9] C. T. Kuo, On $C^{0}$-sufficiency of jets of potential functions, Topology, 8 (1969), 167-171.

[10] M. Lejeune and B. Teissier, Clôture intégrale des idéaux et equisingularité, Centre de Mathématiques, Université Scientifique et Médicale de Grenoble, 1974.

[11] B. Lichtin, Estimation of Łojasiewicz exponents and Newton polygons, Invent. Math., 64 (1981), 417-429.

[12] S. Łojasiewicz, Ensembles semi-analytiques, IHES., 1965.

[13] M. Oka, Non-degenerate complete intersection singularity, Actualités Math., Hermann, Paris, 1997.

[14] M. J. Saia, The integral closure of ideals and the Newton filtration, J. Algebraic Geom., 5 (1996), $1-11$.

[15] B. Teissier, Monômes, volumes et multiplicités, Introduction à la théorie des singularités, II, Travaux en Cours, 37, Hermann, 1988, 127-141.

[16] B. Teissier, Cycles évanescents, sections planes et conditions of Whitney, Singularités à Cargèse (Rencontre Singularités Géom. Anal., Inst. Études Sci., Cargèse, 1972), Astérisque, Nos. 7 et 8, Soc. Math. France, 1973, 285-362.

[17] A. N. Varchenko, Zeta-function of monodromy and Newton's diagram, Invent. Math., 37 (1976), 253-262.

[18] E. Yoshinaga, Topologically principal part of analytic functions, Trans. Amer. Math. Soc., 314 (2) (1989), 803-814.

\section{Carles Bivì̀-Ausina}

Departament de Geometria i Topologia

Universitat de València

Campus de Burjassot

46100 Burjassot (València)

Spain

E-mail: Carles.Bivia@uv.es 